\title{
Aggregation of Consumers Participation in the Ramping of a Demand Response Event
}

\author{
Omid Abrishambaf, Pedro Faria, Zita Vale \\ Polytechnic of Porto (IPP), Porto, Portugal \\ ombaf@isep.ipp.pt,pnf@isep.ipp.pt, zav@isep.ipp.pt
}

\begin{abstract}
As the global population is daily soaring, the need for electrical energy is also increasing. This makes the role of the power distribution network more tangible, as the efficiency of all sectors should be increased. The need for smart management and strategic planning, such as demand response programs are obvious in the context. This paper proposes an aggregator model that employs DR programs for managing the network balance. In this model, a specific analysis has been provided for the ramp period and demand response timeline to show the financial behaviors of the aggregator. In the case study of the paper, two demand response events are proposed using actual consumption profiles and a cost comparison has been presented using various pricing schemes. The results remark the costs related to the ramp period before the event and show how such costs are important in daily electricity expenses of the aggregator model.
\end{abstract}

Index Terms-Demand Response, Aggregator, Ramp Period, Remuneration, Cost Comparison.

\section{INTRODUCTION}

Daily increment of electrical energy consumption worldwide leads to have a lot of environmental problems [1]. In the past decade, employing Distributed Renewable Energy Resources (DRERs) has been significantly increased, as they are eco-friendly and zero-emission in terms of greenhouse gases [2]. Future power distribution networks without any distributed resources are incomplete [3]. However, while a huge RERs are integrated into the current form of electricity networks, network management would be unstable, as such resources have a lot of variation in generation rate [4].

To overcome these kinds of issues, several solutions have been proposed in the literature, such as using microgrid and smart grids [5]. However, these solutions are not in the frame of a concept anymore, and currently, they become a reality in the current context of the power system. The use of smart grids and microgrids relieve the current hierarchical structure of the power system and move towards the transactive energy system and decentralized management of the network [6]. In this context, Demand Response (DR) programs are considered as a solution to mitigate network congestions and instabilities [7]. DR program is defined as altering the consumption patterns of the end-users in response to the incentive paid by a network player [8]. DR programs are classified into two main categories [9]: incentive-based, including Direct Load Control (DLC), Emergency Demand Response Service (EDRS), Interruptible Demand Response Program (IDRP), etc. And price-based programs including Critical Peak Pricing (CPP), Time-Of-Use (TOU), Real-Time Pricing (RTP), etc. Each program has its own specifications and characteristics. As an example, ramp period in each program is different and depends on the technical or economic features of the network. Furthermore, each program has a minimum reduction capacity that makes small-and medium scales consumers incapable to participate directly in such programs [10]. In this situation, aggregator can be considered as a third-party entity that deals with all small-and medium scales consumers to aggregate and participate them in the electricity markets as a unique resource [11]. However, all aggregator models and employed DR programs should well-investigated and surveyed in term of economic features to validate their performance and proves that they are cost-effective.

This paper presents an aggregator model that uses several DR programs to manage a local network, with a specific look on the ramp period and DR timeline. The proposed model demonstrates the role of aggregator in the electricity network and then provides a financial analysis on DR remunerations and electricity costs of the aggregator. There are several similar works in the literature. In [12], the authors presented a precise vision to the ramp period in an aggregator using a laboratory demonstration. However, they did not provide a discussion for remuneration payments and aggregator's costs during the ramp period and the DR event itself. In [13], an analysis of DR programs for consumers equipped with smart metering was presented, that aggregator implements DR programs based on near real-time information. However, the main contribution of this paper is to show financial behaviors and cost comparisons in a DR aggregator model by a specific focus on the ramp period's costs in DR events using various pricing schemes.

After this introductory section, Section 2 proposes the architecture of the aggregator model and the DR program characteristics. In Section 3, a case study is presented to survey the financial behaviors and economic aspects of the model, and, its results are shown in Section 4. Finally, the main conclusions of the paper are demonstrated in Section 5.

The present work was done and funded in the scope of the following project: Eco Rural IoT project funded by TETRAMAX-VALUECHAINTTX-1, CEECIND/02887/2017 and UID/EEA/00760/2019 funded by FEDER Funds through COMPETE program and by National Funds through FCT. 


\section{Methodology DescriPtion}

This section proposes the architecture of the developed model into two subsections. In the first subsections, the nature of the aggregator model is presented with a specific focus on the ramping of DR programs and how aggregator deals with the programs during this period. In the second subsection, the features and characteristics of the suggested DR programs are briefly described.

\section{A. DR Ramping in Aggregator}

As has been addressed in the literature, the aggregator is considered as a third-party entity between the consumers and upstream level players of the network. This entity gathers all small and medium scales electricity customers that somehow tend to participate in the electricity market. Some customers are equipped with RERs, so they would like to sell their energy surplus to the grid, some others attempt to participate in DR programs by managing and reducing their consumption.

In this model, the responsibility of the aggregator is to deal with DR resources to gather and participate them in the electricity markets. For this purpose, this third-party entity has to establish contracts with its clients who intend to participate in the DR program. In such contracts, clients give authorization to the aggregator to manage a part of their consumption, and in exchange, clients will receive financial incentives based on the contractual parameters. In this regard, the aggregator network should be equipped with a power full base for the communication infrastructure. All the network management scenarios, such as DR programs, are dependent on these kinds of infrastructure.

On one hand, the aggregator is in touch with the network players at the upstream level, such as ISO, DSO, or market operators. On the other hand, the aggregator communicates with the contracted end-users on the demand side. Suppose that due to some technical or economic instabilities in the network, ISO or DSO notifies the aggregator to apply for DR programs. Then, the aggregator processes the DR event and transmits the DR request to its consumers. In this step, consumers reply with their preferences to participate in the event or not. However, in some programs, like DLC, there is no need for consumer's permission, and it is mandatory. This is dependent on the type of contract that has been established between the consumer and the aggregator. This leads to having several iterations of DR requests from aggregator to the consumers, as the aggregator cannot anticipate the response of consumers. This procedure continues until the aggregator reaches a pre-defined reduction baseline that fulfills the requirements of upstream level network players.

While ISO or DSO notifies the aggregator to apply for DR programs, a huge amount of data and information will be dispatched between these entities, such as starting point, amount of reduction, duration of the event, etc. In this regard, while the aggregator is aware of the event's schedule, it should notify the consumers in advance to reach the reduction baseline in the starting point. This is known as ramp period, which is referred to as the time the aggregator offers to its consumers to reach the contractual DR reduction baseline. Fig. 1 illustrates the ramp period's timeline used by aggregator.

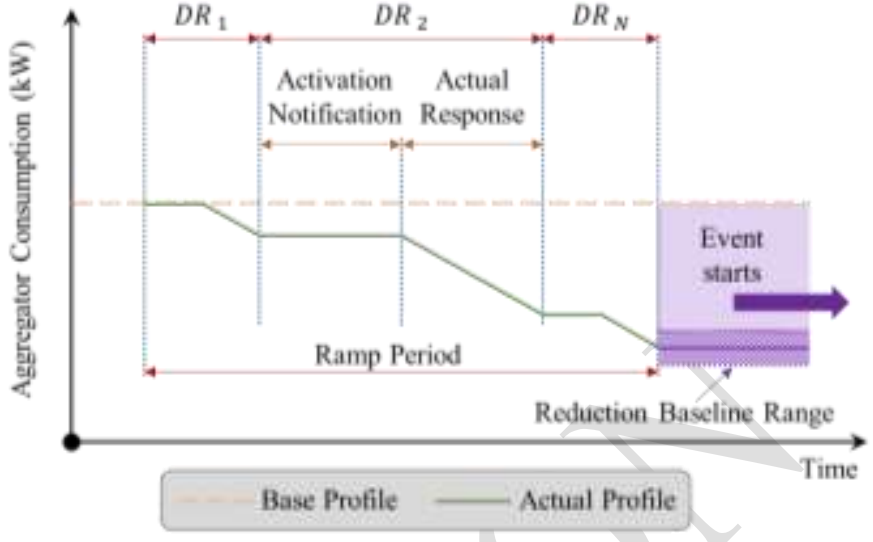

Figure 1. Demand response timeline in the proposed aggregator model.

As Fig. 1 shows, during the ramp period, the aggregator attempts to reach the desired amount of reduction, by notifying the associated consumers for the reduction request. After then, consumers have an activation notification period, which is the time that they can respond to the request with OPT-IN or OPT-OUT. If they are OPT-IN, the aggregator will dedicate the actual response period to them for the load reduction process. During the ramp period, the aggregator continuously evaluates the available reduction capacity. if this capacity is in the range of reduction baseline, the aggregator stops transmitting DR requests to its customers, and it will be ready to start the event. This process is also shown in Fig. 2 in the frame of a flowchart. When the DR event is begun, the timeline enters to a sustained response period. In this period, all consumers who participated in the DR event must keep their committed level of reduction until the end of the event. During all these periods (i.e. ramp period and sustained response period), DR participants convey the real-time consumption profile and other related information to the aggregator.

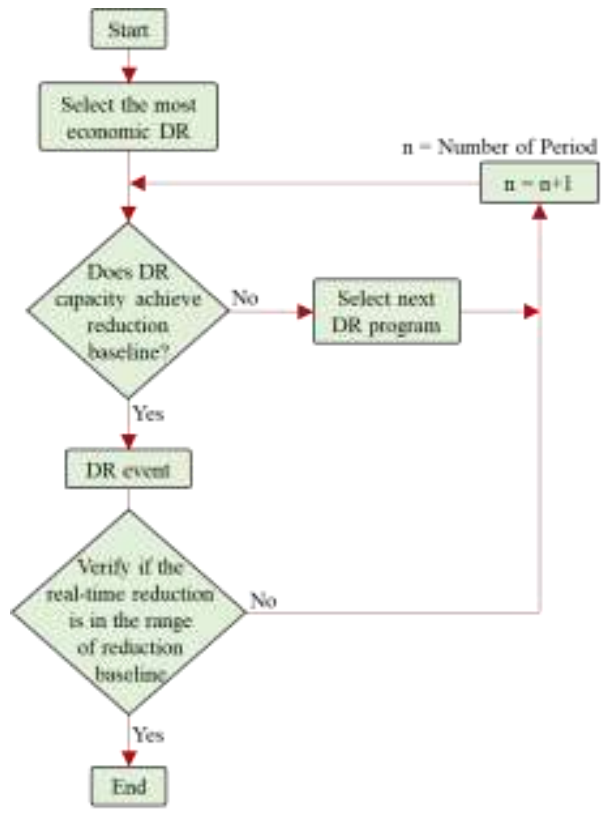

Figure 2. Verification process of reduction baseline during the ramp period and demand response event. 
As it is clear in Fig. 2, aggregator always (in both ramp period and sustained response period) checks the real-time reduction capacity, to verify if it is in the range of reduction baseline. If the real-time reduction goes into a warning range (out of range of reduction baseline), the aggregator immediately applies for some fast-acting DR programs. The flowchart shown in Fig. 2 can be implemented in a controller unit of aggregator through several pre-defined rules to select the most optimal and economic DR programs concerning the remuneration costs and other related expenses.

\section{B. 2.2. DR Program characteristics}

This subsection explains the DR programs that are proposed for the aggregator. Typically, short and real-time DR is the most applicable programs that are used by the aggregator or any other network operator. In this context, incentive-based DR programs are is a nice target to focus on, as they are mostly being applied near to real-time (from a month in advance to 15 minutes [14]). Also, as the focus of this paper is to study the economic behavior and financial survey of aggregator during the ramp period, incentive-based DR programs are interesting to be discussed and applied. For this purpose, Table 1 demonstrates the DR programs that are proposed to be used by the aggregator. These programs have been developed by the authors in the scope of their previous works, and only the most relevant information is mentioned in this section. More information is available on [15].

TABLE I. DEMAND RESPONSE CONTRACTS FEATURES

\begin{tabular}{|c|c|c|c|}
\hline Name & Trigger Signal & Remuneration & Customer Response \\
\hline $\begin{array}{l}\text { DLC } \\
\text { T1 }\end{array}$ & $\begin{array}{l}\text { Reference signal to } \\
\text { the device }\end{array}$ & $\begin{array}{l}\text { Power tariff } \\
\text { discount }\end{array}$ & $\begin{array}{l}\text { Contractual number } \\
\text { of events per month }\end{array}$ \\
\hline $\begin{array}{l}\text { DLC } \\
\text { T2 }\end{array}$ & $\begin{array}{l}\text { Reference signal to } \\
\text { the device }\end{array}$ & $\begin{array}{c}\text { Cost } / \mathrm{kWh} \\
\text { reduced }\end{array}$ & $\begin{array}{l}\text { Actual } \mathrm{kWh} \\
\text { reduction }\end{array}$ \\
\hline IDRP & $\begin{array}{c}\text { Reduction } \\
\text { notification to a } \\
\text { local controller }\end{array}$ & $\begin{array}{l}\text { Cost } / \mathrm{kWh} \\
\text { reduced }\end{array}$ & $\begin{array}{l}\text { Real-Time } \\
\text { consumption }\end{array}$ \\
\hline EDRP & $\begin{array}{c}\text { Actual consumption } \\
\text { level notification }\end{array}$ & $\begin{array}{c}\text { Cost } / \mathrm{kWh} \\
\text { reduced }\end{array}$ & $\begin{array}{l}\text { Actual } \mathrm{kWh} \\
\text { reduction }\end{array}$ \\
\hline
\end{tabular}

As Table 1 shows, DLC T1 is the first suggested DR contract that consumers can establish with the aggregator. In this program, the activation notification would be transmitted directly to the associated device to reduce/increase the consumption. In exchange, consumers will receive a power tariff discount for consumption reduction. In DLC T2, the activation notification signal is similar to DLC T1; However, consumers have to transmit their actual consumption reduction to the aggregator and will receive incentives according to the reduction. In the IDRP contact, the activation notification signal is being transmitted to a local controller on the enduser's side. In the same contract, consumers have to send their real-time consumption level to the aggregator, and in exchange, they will receive incentives for the reduced consumption. The last contract is the EDRP, which in this model is used in the emergency situation while some consumers OPT-OUT during the event, so the aggregator can maintain the committed reduction level.

\section{CASE STUDY}

This section describes a developed case study to validate the performance of the system and survey the financial aspect of the proposed aggregator model. In fact, this case study is based on the author's previous work [12], and the aggregator network configuration has been adapted from the same work. The main purpose of this case study is to survey the economic aspects of the aggregator while it applies to DR programs, with a specific focus on the ramp period. For this purpose, it is considered that the aggregator is responsible for electricity management of a small community of consumers that contains 27 residential buildings. All these residential consumers have a direct contract with the aggregator for DR programs. Also, in this case, study, it is considered that the aggregator buys energy from the electricity markets and sells to its consumers with a certain rate of price in order to gain financial profits. Fig. 3 illustrates the day-ahead consumption profile and available DR resources considered for the aggregator network. The data shown in Fig. 3 are for a random winter day with 15 minutes time interval that has been adapted from the author's previous works [12]. The origin of these data is related to the implementation of an intelligent energy management system in two small cities in Portugal, as referred in [12]. The difference between the total consumption profile and total DR capacity in Fig. 3 is related to the uncontrollable part of consumption that aggregator is not able to manage.

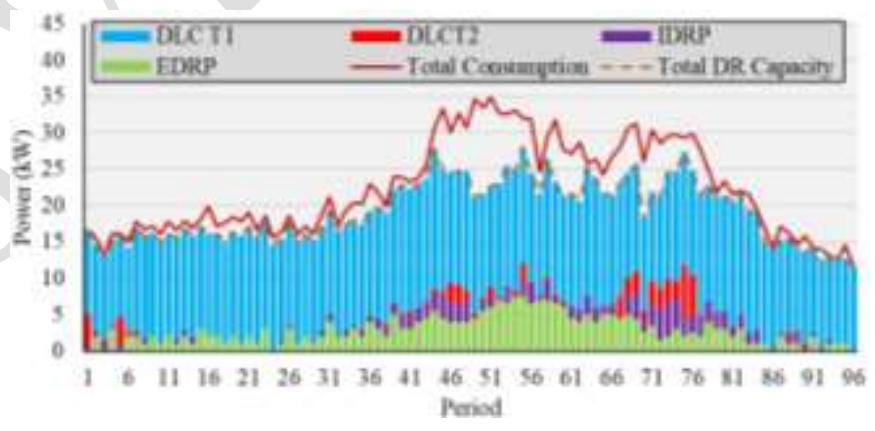

Figure 3. Consumption profile and stacked DR resources capacity in the aggregator network.

Furthermore, Fig. 4 illustrates the proposed DR events to be applied in the aggregator network. The reason for applying these DR events could be a technical fault or any other economic effects in the main grid. In this case study, three scenarios are considered for the aggregator network and survey the financial incomes and outcomes of the aggregator.

In scenario (A) only DR event 1 is being applied in the network, which aggregator has to keep the consumption rate on $20 \mathrm{~kW}$ for two hours starting from noon. In scenario (B), only DR event 2 will be applied, to keep the consumption level on $20 \mathrm{~kW}$ for 2 hours and 30 minutes starting from 4:30 PM. Finally, in scenario (C), it would be a combination of scenario (A) and (B), which is to apply both DR events in the aggregator network. As Fig. 4 shows, the ramp period in DR event 1 is considered as 1 hour, and in DR event 2 is 30 minutes. Fig. 5 demonstrates linear costs considered as a remuneration rate for each DR contract. In fact, aggregator takes these linear costs into the account to start applying the cheapest program in each event. Furthermore, if some 
programs are being activated in the first event, they will not be considered in the second event, which is the main point in scenario $\mathrm{C}$ to compare the financial results.

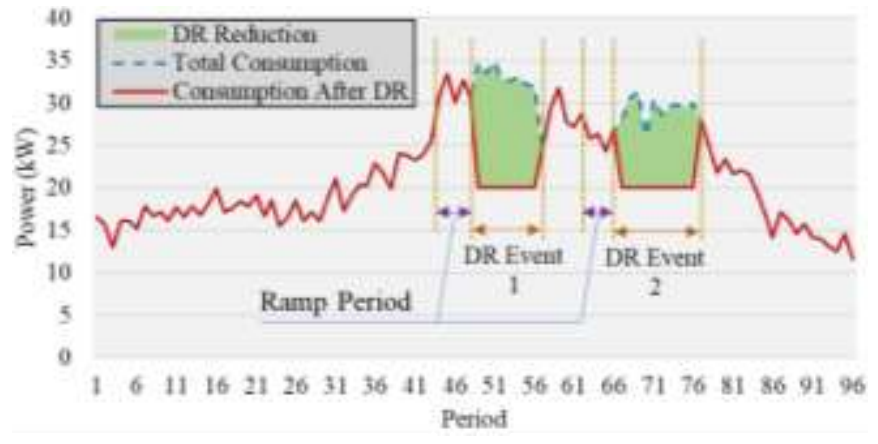

Figure 4. Applied DR events in the aggregator network.

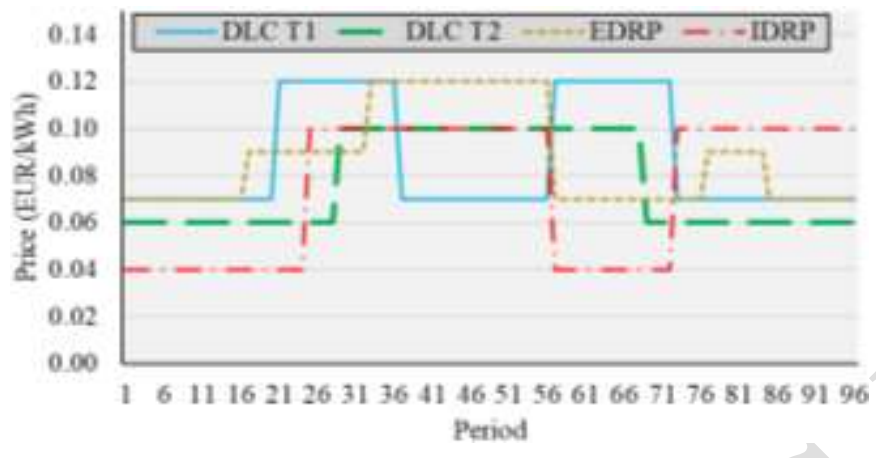

Figure 5. Linear remuneration costs of aggregator for DR programs.

In this case study, three pricing schemes have been presumed for the aggregator to buy and sell electricity. In fact, the aggregator purchases electricity from the markets based on the RTP scheme (red line in Fig. 6) and sells to the consumers based on the TOU tariff (green dashed line in Fig. 6) or a simple tariff (blue dashed line in Fig. 6). These TOU and simple tariffs are actual pricing schemes that are currently utilized at the incumbent Portuguese electricity retailer (www.edp.pt). Moreover, the RTP scheme is adapted from the Iberian electricity market (www.omie.es).

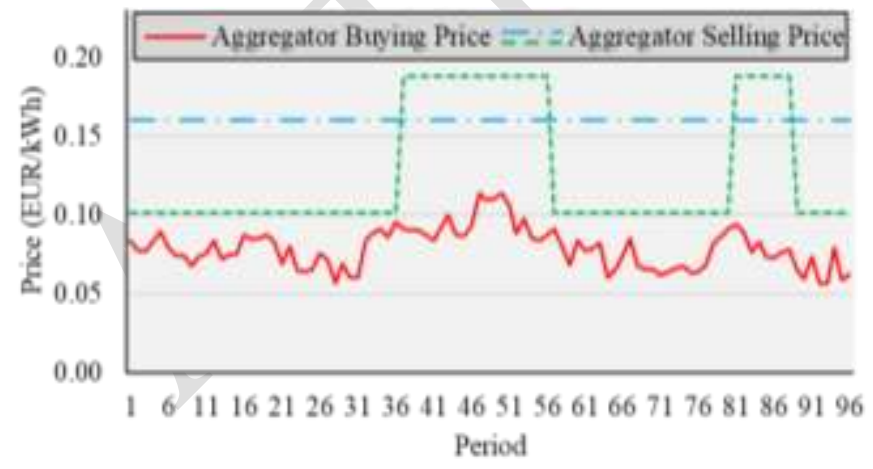

Figure 6. Pricing schemes of energy transactions in the aggregator network.

In the typical electricity network, TOU and simple tariffs are long-term pricing schemes that are always higher than market prices. This enables the aggregators or any retailer to gain financial benefits through selling energy to the end-users.

\section{RESULTS}

This section presents the obtained results of the case study, while the DR events have been applied in the aggregator network. In this context, during the ramp period and the event itself, the aggregator uses the flowchart shown in Fig. 2 as well as the linear remuneration costs illustrated in Fig. 5, to apply the most economic DR resources first and move towards the most expensive resources in terms of remuneration. This process is to be continued until the aggregator reaches to the desired baseline reduction. As it was described in the case study, the reduction baseline in both events was set on $20 \mathrm{~kW}$. This leads to having a reduction of 12 to $15 \mathrm{~kW}$ in scenario (A), and around $10 \mathrm{~kW}$ in scenario (B). According to the obtained results, during the DR event 1 , in scenario (A), DLC T1 has a high capacity of reduction with a lower rate of remuneration. So, it covered all the required reduction for event 1 . However, in DR event 2, the DLC T1 would be no more available to the aggregator as it has been already used in the first event. On the other hand, the remuneration rate of DLC T1 is higher during the second event, therefore, the aggregator moves towards the other economic DR resources with a lower rate of remuneration considering their reduction capacity. This leads to having DLC T2 and IDRP during the DR event 2, and in some cases, EDRP appears to cover the required reduction. Table 2 demonstrates the cost calculation and comparison between all scenarios all day. In fact, Table 2 shows detailed information regarding the costs of the aggregator in various situations, such as with or without implementing DR programs. The income in Table 2 stands for the profits that aggregator receives from selling electricity to the consumers. The outcome in the same table is the expenses that aggregator pay to purchase electricity from the market, and finally, the sum column represents the result of financial transactions. All the costs shown in Table 2 are in EURO.

\section{TABLE II. COSTS COMPARISON IN ALL SCENARIOS OF CASE STUDY}

\begin{tabular}{|c|c|c|c|c|c|}
\hline & & & Income & Outcome & Sum \\
\hline \multicolumn{3}{|c|}{ Daily Energy Cost Before DR } & +70.16 & -43.08 & +27.08 \\
\hline \multirow[t]{2}{*}{$\underset{\mathbf{A}}{\text { Scenario }}$} & $\begin{array}{l}\text { DR } \\
\text { Costs }\end{array}$ & $\begin{array}{c}\text { Ramp } \\
\text { Period } \\
\text { Sustained } \\
\text { Response }\end{array}$ & - & $\begin{array}{l}-0.49 \\
-1.78\end{array}$ & -2.27 \\
\hline & \multicolumn{2}{|c|}{$\begin{array}{c}\text { Daily Energy Costs } \\
\text { After DR }\end{array}$} & +64.05 & -39.88 & +21.90 \\
\hline \multirow[t]{2}{*}{$\begin{array}{c}\text { Scenario } \\
\text { B }\end{array}$} & $\begin{array}{l}\text { DR } \\
\text { Costs }\end{array}$ & $\begin{array}{c}\text { Ramp } \\
\text { Period } \\
\text { Sustained } \\
\text { Response }\end{array}$ & - & $\begin{array}{l}-0.11 \\
-1.53\end{array}$ & -1.64 \\
\hline & \multicolumn{2}{|c|}{$\begin{array}{c}\text { Daily Energy Costs } \\
\text { After DR }\end{array}$} & +67.68 & -41.44 & +24.60 \\
\hline \multirow[t]{2}{*}{$\underset{\mathrm{C}}{\text { Scenario }}$} & $\begin{array}{l}\text { DR } \\
\text { Costs }\end{array}$ & $\begin{array}{c}\text { Ramp } \\
\text { Period } \\
\text { Sustained } \\
\text { Response }\end{array}$ & - & -3.31 & -3.91 \\
\hline & \multicolumn{2}{|c|}{$\begin{array}{c}\text { Daily Energy Costs } \\
\text { After DR }\end{array}$} & +61.57 & -38.23 & +19.43 \\
\hline
\end{tabular}

In Table 2, the daily income costs in all scenarios have been calculated based on the TOU tariff, and the outcome 
costs have calculated according to the RTP scheme shown in Fig. 6. In fact, the cost of sustained response in Table 2 is related to remuneration that aggregator pays to the consumers during the DR event. Also, during the ramp period, consumers start reducing their consumption, so they expect to receive remuneration for their reduction. Therefore, aggregators or any other DR program manager should consider the costs related to the ramp period, as Table 2 shows in this paper. Focusing on total daily costs, Fig. 7 represents the total accumulated costs of aggregator during scenario $\mathrm{C}$, where both DR events have been applied. In Fig. 7 - (A), the income and outcome of aggregator are compared with and without DR events. Besides, Fig. 7 - (B) shows the income of aggregator in scenario (C) while various pricing schemes are applied to the consumers. All the employed tariffs for calculating the results shown in Fig. 7 - (B), are previously represented in Fig. 6, while some coefficients are applied in this regard.

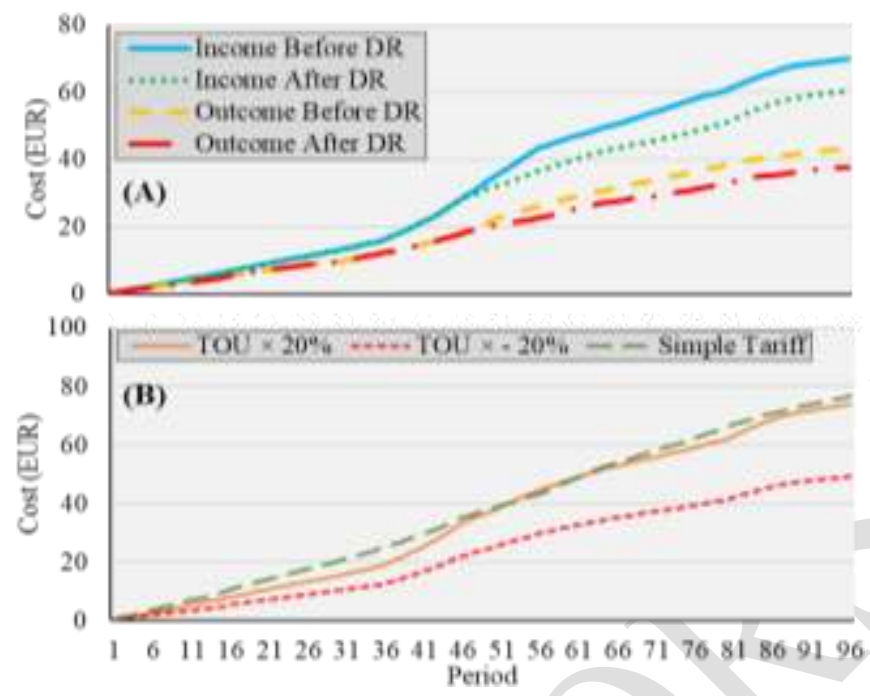

Figure 7. Accumulated costs comparison during scenario (C): (A) daily income and outcome costs, (B) income cost using various pricing schemes.

The main purpose of the results shown on Table 2 and Fig. 7 , is to demonstrate the aggregator's cost during the ramp period and DR program itself. In this case study, only 27 consumers are considered in the aggregator network, however, the model has capability of implementation for a larger scale with a huge number of consumers. Although in this model, the aggregator's costs during the ramp period are not significant for these 27 consumers; in a larger scale, this little cost becomes remarkable and it causes a huge cost variation between what it is expected and what occurs in actual cases

\section{CONCLUSIONS}

This paper presented an aggregator model that utilized several incentive-based demand response programs to manage a local network. In this model, a precise look was given to the ramp period of demand response and its timeline in the scope of financial analysis and cost comparison. In the case study of the paper, several scenarios were implemented using real consumption profiles and demand response data. The results of the case study provided the daily costs of the aggregator with and without demand response programs, using remuneration tariffs and various electricity pricing schemes. Also, the costs related to the ramp period was demonstrated. Most of the aggregator models in the current literature consider that the demand response's costs are only related to starting and ending points of the event. However, from the results of this paper, it can be concluded that the aggregator will never reach the desire reduction level immediately, and it requires some time to reach to reduction baseline. On the other hand, consumers would like to receive incentive payment as soon as they've started the consumption reduction. Therefore, the costs related to the periods prior to the event, such as ramp period, should be always considered in all aggregator models as these costs are a part of DR remuneration calculations.

\section{REFERENCES}

[1] H. Zou, B. Luan, X. Zheng, and J. Huang, "The effect of increasing block pricing on urban households' electricity consumption: Evidence from difference-in-differences models," J. Clean. Prod., vol. 257, p. 120498, Jun. 2020.

[2] L. Tjørring, C. L. Jensen, L. G. Hansen, and L. M. Andersen, "Increasing the flexibility of electricity consumption in private households: Does gender matter?," Energy Policy, vol. 118, pp. 9-18, Jul. 2018.

[3] S. Haghifam, M. Dadashi, K. Zare, and H. Seyedi, "Optimal operation of smart distribution networks in the presence of demand response aggregators and microgrid owners: A multi follower Bi-Level approach," Sustain. Cities Soc., vol. 55, p. 102033, Apr. 2020.

[4] X. He, N. Keyaerts, I. Azevedo, L. Meeus, L. Hancher, and J.-M. Glachant, "How to engage consumers in demand response: A contract perspective," Util. Policy, vol. 27, pp. 108-122, Dec. 2013.

[5] M. Mohammadi, Y. Noorollahi, B. Mohammadi-ivatloo, M. Hosseinzadeh, H. Yousefi, and S. T. Khorasani, "Optimal management of energy hubs and smart energy hubs - A review," Renew. Sustain. Energy Rev., vol. 89, pp. 33-50, Jun. 2018.

[6] O. Abrishambaf, F. Lezama, P. Faria, and Z. Vale, "Towards transactive energy systems: An analysis on current trends," Energy Strateg. Rev., vol. 26, p. 100418, Nov. 2019.

[7] F. Wang et al., "Smart Households' Aggregated Capacity Forecasting for Load Aggregators Under Incentive-Based Demand Response Programs," IEEE Trans. Ind. Appl., vol. 56, no. 2, pp. 1086-1097, Mar. 2020.

[8] M. Khorram, P. Faria, O. Abrishambaf, and Z. Vale, "Demand Response Implementation in an Optimization Based SCADA Model Under Real-Time Pricing Schemes," in International Symposium on Distributed Computing and Artificial Intelligence, 2019, pp. 21-29.

[9] R. Alasseri, T. J. Rao, and K. J. Sreekanth, "Conceptual framework for introducing incentive-based demand response programs for retail electricity markets," Energy Strateg. Rev., vol. 19, pp. 44-62, Jan. 2018.

[10] P. Siano, "Demand response and smart grids-A survey," Renew. Sustain. Energy Rev., vol. 30, pp. 461-478, Feb. 2014.

[11] O. Abrishambaf, P. Faria, and Z. Vale, "SCADA Office Building Implementation in the Context of an Aggregator," in 2018 IEEE 16th International Conference on Industrial Informatics (INDIN), 2018, pp. 984-989.

[12] O. Abrishambaf, P. Faria, and Z. Vale, "Ramping of Demand Response Event with Deploying Distinct Programs by an Aggregator," Energies, vol. 13 , no. 6 , p. 1389 , Mar. 2020.

[13] H. Zhong, L. Xie, and Q. Xia, "Coupon Incentive-Based Demand Response: Theory and Case Study," IEEE Trans. Power Syst., vol. 28, no. 2, pp. 1266-1276, May 2013.

[14] P. Faria and Z. Vale, "A Demand Response Approach to Scheduling Constrained Load Shifting," Energies, vol. 12, no. 9, p. 1752, May 2019.

[15] O. Abrishambaf, P. Faria, and Z. Vale, "Participation of a Smart Community of Consumers in Demand Response Programs," in 2018 Clemson University Power Systems Conference (PSC), 2018, pp. 1-5. 